\title{
A Study of the Optical Characteristics of CR-39 Nuclear Track Detector Using Ultraviolet
} Irradiation

\author{
Tayseer I. Al-Nagaar ${ }^{a, b, d}$ and Doaa H. Shabaan ${ }^{a, c^{*}}$ \\ (a) Department of Physics, College of Women, for Art, Science and Education, Ain Shams University, Cairo, Egypt. \\ ${ }^{(b)}$ Department of Physics, Faculty of Arts and Sciences, Najran University,Najran, Saudi Arabia. \\ (c) Physics Department, University collage of Samtah, Jazan University, Jazan, KSA. \\ (d) Unit of Radiation protection, Najran University, Najran1101, Saudi Arabia.
}

\begin{tabular}{|c|c|}
\hline ARTICLE INFO & ABSTRACT \\
\hline Article history: & \multirow{9}{*}{$\begin{array}{l}\text { The effect of the UV radiation is studied to investigate the optical properties of } \\
\text { CR-39 polymer. The measurements of FTIR spectroscopy show maintenance of the } \\
\text { main vibratory band resulting from the irradiation times. The optical absorption } \\
\text { spectrum of UV / VS indicates a main alteration in the optical energy gap with } \\
\text { irradiation times. From the results, it was found that increasing the irradiation time } \\
\text { of CR-39 by UV- lamp leads to an increase in the sensitivity of the detector and } \\
\text { improvement of its properties and thus achieve safe use of these detctors. FTIR } \\
\text { spectra shows the conservation of the distinctive spectral peaks related to the } \\
\text { monomer of polymer before and after exposure to different intensities of UV-lamp. } \\
\text { From this results, the CR-39 detector ultraviolet irradiated were conformed to be } \\
\text { very sensitive to UV radiation. }\end{array}$} \\
\hline Received: $1^{\text {st }}$ Feb. 2021 & \\
\hline Accepted: $16^{\text {th }}$ June 2021 & \\
\hline Keywords: & \\
\hline UV-Lamp, & \\
\hline CR-39, & \\
\hline Radiation, & \\
\hline FTIR, & \\
\hline UV visible spectroscopy. & \\
\hline
\end{tabular}

\section{INTRODUCTION}

Nuclear track detectors such as CR-39 is used in various fields such as the manufacture the lenses of glasses and in building as wall. CR-39 detector can also be used to measure radon and neutron dosages [1]. During their use, nuclear path detectors can be detected at temperatures above ambient levels. CR-39 is the nuclear path detector could maintain the dosage information under different environmental conditions. Ultraviolet rays have a very large effect on detector sensitivity. UV lamp exposure increases the volume, total volume and drilling rate of the average path at peaks corresponding to 254 and $350 \mathrm{~nm}$ lines, while decreasing at $300 \mathrm{~nm}$. [2].

The influence of UV- rays- on CR-39 detector has been examined in several works. The CR-39 detector gradually decomposes due to changes in its chemical and physical characteristics and gradually turns yellow when exposed to UV-rays[3]. UV radiation and photons having more energy can have important and profound effects on the properties of path detectors behind electrons or other particles that may not be able to be monitored. The effects detected are restricted to plastics, unless the doses are very high [4]. By breaking chemical bonds in polymers, the molecular chains can be separated, which can occur with less energy transfer than usually required for ionization (2-3 eV vs. 10-15 eV) [5]. UV rays are easily spread through glass and air. Ultra Violet absorption spectral range, UVB (Ultraviolet A has a longer wavelength) and UVC (Ultraviolet B has a shorter wavelength) rays travel through the air and quartz, but get absorbed in regular glass. The reason is that these wavelengths are absorbed by layer of ozone in the higher atmosphere because the solar radiation on Earth's surface is almost free of wavelengths less than 320 nanometers. Ultraviolet rays under $315 \mathrm{~nm}$ is absorbed primarily by the layer of skin. Ultraviolet rays with wavelengths Less than $200 \mathrm{~nm}$ are not simply transferred by air and are typically only found in a space [6,7].

The current work aims at studying the properties of CR-39 (structural and optical) under irradiation of ultraviolet lamp for different exposure times, because polymer detector of CR-39 is used as an UV dosimeter in several areas such as UV-Rays for solar atmospherics, industrial laboratory and UV-radiation measurements 


\section{Practical Analysis}

\subsection{Material and irradiation}

The molecular formula of CR-39 polymer is $\left(\mathrm{C}_{12} \mathrm{H}_{18} \mathrm{O}_{7}\right)$, its density is $1.32 \mathrm{gm} . \mathrm{cm}^{-3}$ and the temperature of glass transition is $85{ }^{\circ} \mathrm{C}$. Sheet created and introduced by Track Systems Ltd. (TASL), Bristol, United Kingdom. Standard a thickness of $600 \mu \mathrm{m}$ was reported. It was split into a size of $1.5 \times 1.5 \mathrm{~cm}^{2}$. UV lamp of $50 \mathrm{~W}$ was used for exposure. In this study, ten samples were exposed to integrated UV lamp in the times varied from 2 to 10 hours. In the UV band, almost the visible spectrum is fully invisible and has a high impedance of corrosion. The system of irradiation contains on UV lamp (FH1200-X) of $220 \mathrm{~W}$ at $220 \mathrm{~V}$. Samples were exposed to a constant irradiation at a space of $70 \mathrm{~cm}$ through a collimator to the UV-lamp source.

\subsection{UV/Vis Absorption}

The alteration in optical absorption of virgin and CR-39 -irradiated samples were executed by Spectra of UV/vis. UV/vis Spectra was achieved by UV/Visible Double Beam spectrometer JASCO V-630.The wavelength range of JASCO technique is $200-1100 \mathrm{~nm}$ at an interval of $1 \mathrm{~nm}$, at ambient circumstances.

\subsection{FTIR- Technique}

FTIR technique can be used for the clarification of functional groups in CR-39 polymer molecules. Within the range of 400 to $4000 \mathrm{~cm}^{-1}$ for wavelength and $4 \mathrm{~cm}^{-1}$ resolution of the Nicolet 6700 spectrometer- thermoscientific, the spectrum of CR-39 samples was achieved. To gain the alteration in the functional groups of CR-39 polymer between the virgin and the irradiated samples with UV lamp these spectra was analyzed.

\section{RESULTS AND DISCUSSION}

In the absorption behavior, the analysis of UV-Visible spectra was conducted for the pristine and UV-irradiated CR-39 samples through elevated to varied UV lamp doses. The effect of UV wavelength (200400nm) on CR-39 samples are obviously appeared by absorption rate in this range of UV-wavelength as shown in Fig.(1), and the absorption rates increased gradually by increasing the irradiation time as $10 \mathrm{~h}>8 \mathrm{~h}>6 \mathrm{~h}>4 \mathrm{~h}$ $>2 \mathrm{~h}>0 \mathrm{~h}$. The peak of UV-Visible deviates from the UV area to the visible area, which is heading to the greater wavelength, for the irradiated samples. This clarified that a significant decrease occurred in optical band gap energy after UV- irradiation, and the expanding of the absorption peak through increasing the exposure time[8].

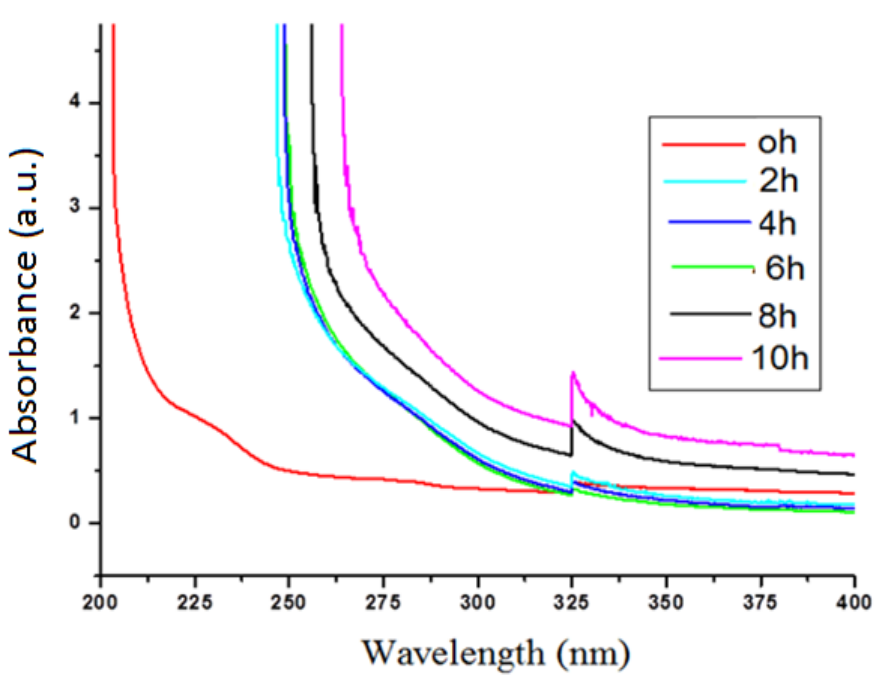

Fig. (1): Absorption process and wavelength for the CR-39 detectors.

Fig. (2) displays the manners of absorption process as a function of the irradiation time on the absorbance spectrum for all the investigated samples at the wavelength varied from $200 \mathrm{~nm}$ to $400 \mathrm{~nm}$. It can be observed from this Figure that there is a linear relationship between the absorption rate and the irradiation time which has a strong coefficient $\left(\mathrm{R}^{2}=0.78\right)$ and that with increasing the irradiation time, the absorption rate increases. This positive relationship demonstrates the effects of radiation on polymeric substances, and this linear relationship represents the probability that alpha particle energy can be measured using CR-39 polymer track detectors using absorbance variance only within this range $[9,10]$.

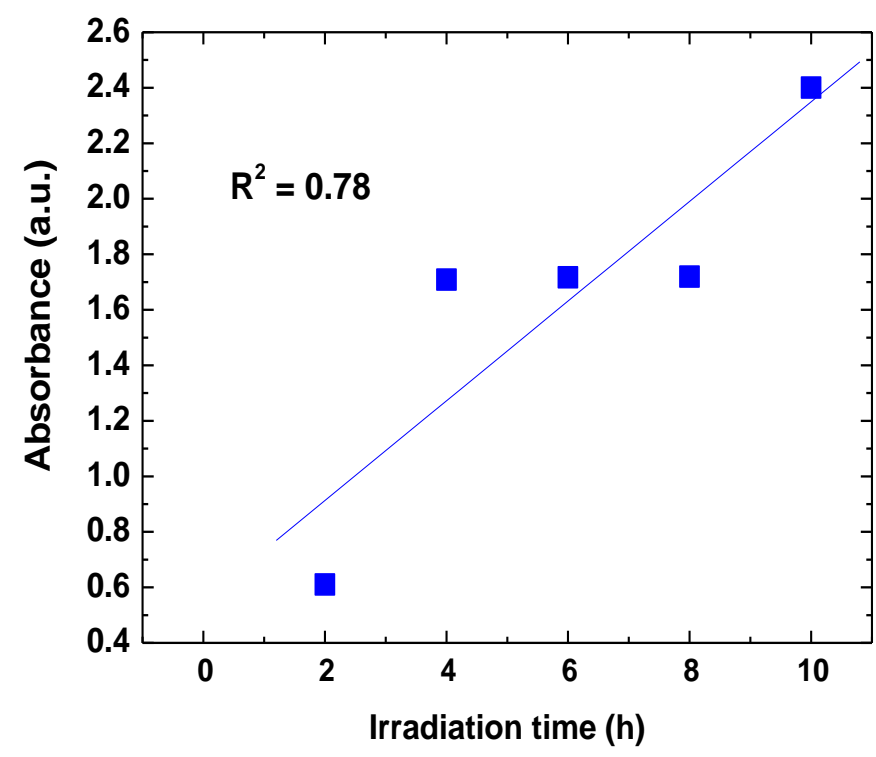

Fig. (2): Absorption rate as function of the UV irradiation time. 


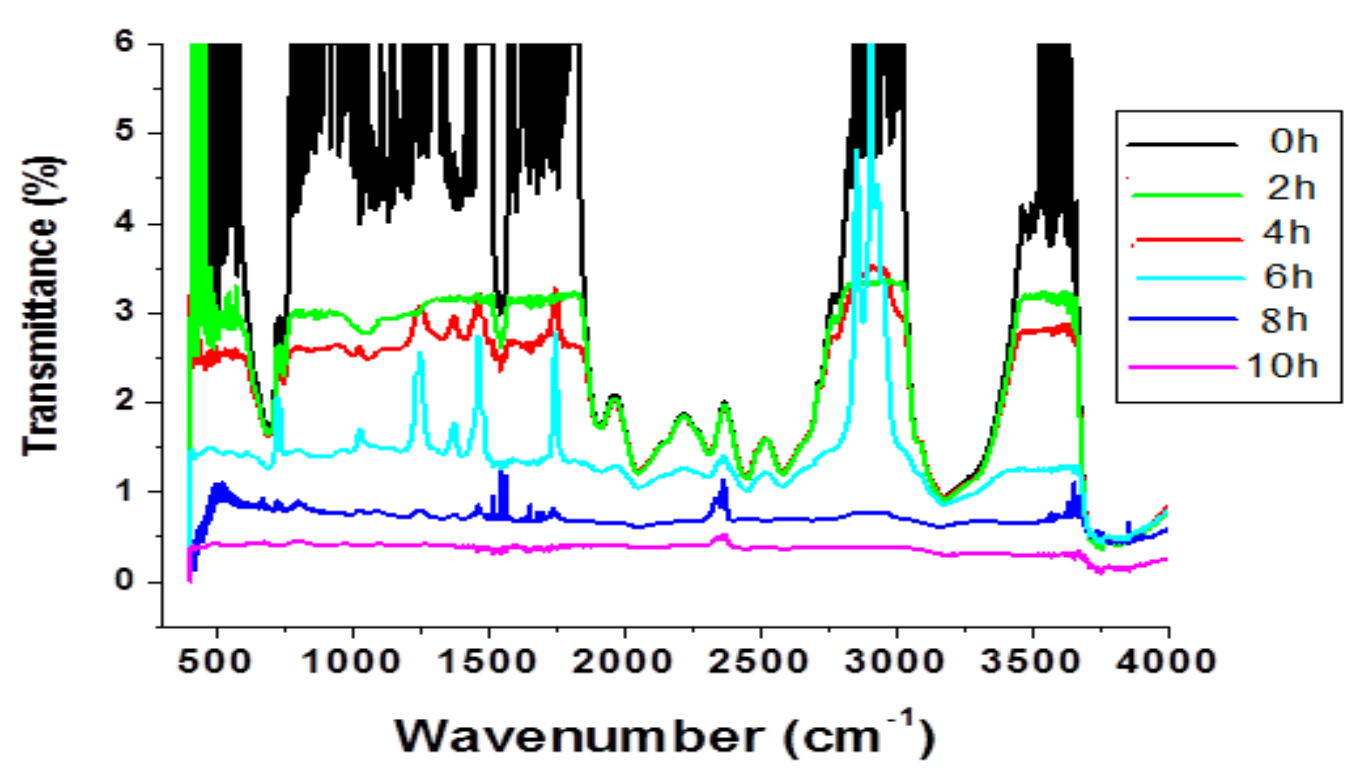

Fig. (3) FTIR spectra of pristine and irradiated CR-39 samples.

Fig.( 3) shows the FTIR spectra which indicates the conservation of the distinctive spectral peaks related to the monomer of polymer before and after exposing to different intensities of UV lamp. Fig. (3) shows the FTIR spectrum of CR-39 in its wavenumber $\left(\mathrm{cm}^{-1}\right)$ and the related significance of the functional groups related to pristine CR-39 sample. It is obvious that the main distinctive peaks of the pristine sample related to obvious functional groups. The registered corresponding band peaks of the sample are at $657 \mathrm{~cm}^{-1}, 1462 \mathrm{~cm}^{-1}$, $1773 \mathrm{~cm}^{-1}, 2348 \mathrm{~cm}^{-1}, 2849 \mathrm{~cm}^{-1}, 3282 \mathrm{~cm}^{-1}$ and 3542 $\mathrm{cm}^{-1}$ which are relevant to the absorption band of $\mathrm{CO}_{2}$, bending vibration related to $\mathrm{CH}_{3}$ and to $\mathrm{CH} 2$ small bands, $\mathrm{C}-\mathrm{O}$ stretch, absorption band of $\mathrm{CO}_{2}, \mathrm{C}-\mathrm{H}$ symmetric stretching of $-\mathrm{CH}_{2}-$, alkynes end group $(\mathrm{R}-\mathrm{C} \equiv \mathrm{C}-\mathrm{H})$ and $\mathrm{OH}$ group and / or adsorbed water respectively $[11,12]$. The bands intensity have been shown to decrease as the irradiation phase increases, which points to the decrease in the unsaturated behavior of polymers. The irradiation time ions in the bands related to $8 \mathrm{~h}$, and $10 \mathrm{~h}$ were found to disappear with increasing radiation dose, this decrease in the intensity of $\mathrm{C}=\mathrm{O}$ band has also been observed [11]. The decrease in peak intensity may be related to the splitting of the carbonate bond chain producing $\mathrm{CO}_{2}$ and carboxylic acids $[13,14,15]$. The relationship between the cleavage of carbonate and the removal of $\mathrm{H}$ from the polymer structure is related to the decrease in intensity that results in the formation of $\mathrm{CO}_{2}$ and-OH [16].

\section{CONCLUSION}

Ultraviolet irradiation investigation of the absorption process is a significant mechanism that relates to the structural variations within the polymer material. The
UV/vis optical absorption spectrum induces a progressive increase in the absorption rate by increasing the irradiation time. FTIR spectra shows the conservation of the distinctive spectral peaks related to the polymer monomer before and after exposure to different intensities of UV lamp. These results indicate that the ultraviolet irradiated CR-39 detectors were conformed to be very sensitive to UV radiation.

\section{REFERENCES}

[1] Sinha Dipak. (2012) Gamma irradiation effects on track properties of PADC-American acrylics track detector, E-Journal of Chemistry, 9(4), 2226-2231.

[2] Babak Jaleh, Parviz Parvin, Kavoos Mirabaszadeh and Mehran Katouzi, (2004) KrF laser irradiation effects on nuclear track recording properties of polycarbonate, Journal of Radiation Measurements, 38, 173 -183.

[3] Hussein, A. Ahmed., Alan, S. Said, Ahmad. and Ari, A. Mohammed. (2015) Measure the bulk etch rate using the time-diameter method, Journal of Materials Sciences and Applications, 6, 286-291.

[4] Fleischer, R.L., Price, P.B. and Walker, R.M. (1975) Nuclear Tracks in Solids: Principles and Application, University of California Press, Berkeley, USA.

[5] Fleischer, R.L. and Bull, M.R.S. (1995).

[6] Walmer Avenue Norfolk, (1992) Ultraviolet Radiation Guide, Navy Environmental Health Center, 2510, 23513-2617.

[7] Biswa Ranjan Das. (2010) UV Radiation Protective Clothing, The Open Textile Journal, 3, 14-21. 
[8] Saad, A.F., Mona, H. Ibraheim and Mona, Hamada. (2019) IR radiation-induced changes in the optical and registration properties of a CR-39 nuclear track detector, Radiation Measurements, 126, 106-129.

[9] Hassan, M. J. A. (2020) Investigating the optical characteristics of SSNTD-LR 115 by using different UV radiation dose, Radiation Measurements, 132, 106-162.

[10] Rammah Y.S, Awad, E.M. (2018). Modifications of the optical properties for DAM-ADC nuclear track detector exposed to alpha particles. Radiation Physics and Chemistry 146, 42-46.

[11] Zaki, M. F., AbdulKader, A. M., Afaf, N., Basma, A. El-Badry. (2013) Surface modification of Makrofol-DE induced by $\alpha$-particles, Philosophical Magazine, 8, 27-33.

[12] El Ghazaly, M. and Hassan, H. E. (2014) Spectroscopic studies on alpha particle-irradiated PADC (CR-39 detector), Results in Physics, 4, 40 43.
[13] Babak, J., Atefeh, N., Nima, S. and Hanieh, N. (2017) Surface properties of UV irradiated CR-39 polymer before and after chemical etching and registration of fingerprints on CR-39, Radiation Measurements, 101, 22-28.

[14]Tayseer, I. AL-Naggar and Doaa H. Shabaan. (2019) Study of the physical and structural properties of CR-39 detector using $\mathrm{Pu}-239$ alpha source, Interciencia Journal, 44(3), 43-56.

[15] Doaa Hassan Shabaan, Tayseer Ibrahim ALNaggar. (2019). Improvement in Optical Properties of Nuclear Track Detector, Nuclear Science. 4(2), 23-26.

[16] Vijay, K., Sonkawade, R.G., Chakarvarti, S. K., Paramjit, S. , Dhaliwal, A. S.(2012). Carbon ion beam induced modifications of optical, structural and chemical properties in PADC and PET polymers. Radiation Physics and Chemistry 81, 652-658. 